\title{
LDPC Codes for Gaussian Broadcast Channels
}

\author{
Peter Berlin \\ EPFL, Lausanne, Switzerland \\ Email: peter.berlin@epfl.ch
}

\author{
Daniela Tuninetti \\ EPFL, Lausanne, Switzerland \\ Email: daniela.tuninetti@epfl.ch
}

\begin{abstract}
We study coding over a class of two-user broadcast channels with additive white Gaussian noise and fading known at the receivers only. Joint decoding of low-density parity-check codes is analyzed. The message update rule at the mapping node linking the users' codes is derived and is shown to exhibit an interesting soft interference cancellation property. Good degree distributions are found using the differential evolution optimization technique and extrinsic information transfer analysis. The corresponding codes have rates close to the boundary of the achievable region for binary constrained input channels, both with and without fading. Simulation results for moderate blocklength show that the optimized codes operate within $1 \mathrm{~dB}$ of their thresholds.
\end{abstract}

\section{INTRODUCTION}

A Broadcast Channel (BC) is a multi-terminal network that consists of one sender and multiple receivers. The information theoretic capacity region of general BCs is still unknown 30 years after Cover's original problem formulation [1]. In this work we restrict our attention to two-user BCs with ergodic fading and additive white Gaussian noise. We assume that the fading process is known at the receivers but unknown at the transmitter. Tuninetti et al. [2] showed that, as opposed to the degraded case [3], stripping at the best receiver might incur performance degradation. Instead, the two superimposed codewords must be jointly decoded, like in the multiple access case [3]. Our goal is to determine code pairs that perform close to the Shannon limit when superimposed at the transmitter and jointly decoded at one of the receivers only.

In our search for good codes, we concentrate on LDPC (lowdensity parity-check) codes. We are encouraged by recent results showing that LDPC codes achieve capacity for binary erasure channels [4] and achieve reliable transmission at an SNR (signal-to-noise ratio) extremely close to the Shannon limit for memoryless binary-input Gaussian channels in the unfaded case [5], in the Rayleigh fading case [6], in the unfaded multiple access case [7] and in the multi-antenna case [8].

In order to design the degree distributions of "good" LDPC codes, we first derive the stability condition for iterative decoding on the considered broadcast channel model and we check the candidate codes against this condition in order to ensure vanishing bit-error rate. We then apply the extrinsic information transfer (EXIT) analysis [9] to determine the convergence property of the candidate LDPC ensemble under iterative decoding. Finally, we optimize the degree distributions based on the differential evolution algorithm [5]. For the derived codes, we further investigate the bit-error rate (BER) performance for finite blocklength.

In deriving a multiuser version of the message passing decoding algorithm, we show that the node linking the users' codes exhibits an interesting soft interference cancellation property.
The update rule at such a node amounts to stripping from the received signal the contribution from the other user's codeword.

The rest of the paper is organized as follows. In Section II we briefly review LDPC codes and the message passing decoding algorithm; in Section III we describe the model for the two-user fading Gaussian BC, we derive an achievable region when the codes are restricted to be binary, we derive the multiuser version of the message passing decoding algorithm and we show that there exist nodes in the graph that perform soft interference cancellation; in Section IV we derive the stability condition, we describe the optimization technique and we comment on the found codes. Finally, in Section V we point out our conclusions.

\section{BACKGROUND MATERIAL}

In the following we shall indicate with $\mathcal{N}_{R}(\boldsymbol{\mu}, \mathbf{K})$ (resp. $\mathcal{N}_{C}(\boldsymbol{\mu}, \mathbf{K})$ ) a vector of real (resp. proper complex) Gaussian random variables with mean $\boldsymbol{\mu}$ and covariance matrix $\mathbf{K}$, with $\chi_{\{\varepsilon\}}$ the indicator function of the event $\mathcal{E}$, with $\mathrm{E}_{X}$ the statistical average with respect to the distribution of the random variable $X$ and with $f^{-1}(\cdot)$ the inverse of the function $f(\cdot)$.

LDPC codes [10] are a class of random-like linear binary codes with at least one sparse parity-check matrix. They are characterized by the variable and check node degree distributions specified by the polynomials

$$
\lambda(x)=\sum_{i=2}^{d_{\mathrm{v}}} \lambda_{i} x^{i-1} \quad, \quad \rho(x)=\sum_{i=2}^{d_{\mathrm{c}}} \rho_{i} x^{i-1}
$$

where $d_{\mathrm{v}}$ and $d_{\mathrm{c}}$ are, respectively, the maximum variable and check node degree and where the non-negative coefficients $\lambda_{i}$ and $\rho_{i}$ represent, respectively, the fraction of edges emanating from a variable and check node of degree $i$. The code design rate is $r=1-\left(\int_{0}^{1} \rho(x) \mathrm{d} x\right) /\left(\int_{0}^{1} \lambda(x) \mathrm{d} x\right)$.

LDPC codes are efficiently decoded using the sum-product algorithm [11], which is known in this context as the messagepassing decoding algorithm [4]. In the rest of the section we review the LDPC message-passing decoding algorithm for fading single-user Gaussian channels with the primary intention to introduce the notation and to ease the extension to the multiple user case.

A complex-valued single-user fading Gaussian channel is described by $Y=A X+N_{y}$ where $A$ is an ergodic fading process perfectly known at the receiver but unknown at the transmitter, $N_{y} \sim \mathcal{N}_{C}\left(0, N_{0}\right)$ is the additive white Gaussian noise and $X$ is the useful signal subject to the power constraint $\mathrm{E}\left[|X|^{2}\right] \leq P$. Let $\beta=P / N_{0}$ be the average transmit SNR. The channel capacity $C_{A}(\beta)$, in bits per channel use, is

$$
C_{A}(\beta)=\mathrm{E}_{A}\left[\log _{2}\left(1+|A|^{2} \beta\right)\right]
$$

and it is achieved by $X \sim \mathcal{N}_{C}(0, P)$ [3]. If the input alphabet is constrained to be binary, then the largest achievable mutual information is $J_{A}(\beta)=\mathrm{E}_{A}\left[J\left(|A|^{2} \beta\right)\right]$ where the function $J(x)$, for $x \geq 0$, is defined as

$$
J(x)=\mathrm{E}_{M \sim \mathcal{N}_{R}(0,1 / 2)}\left[1-\log _{2}\left(1+\mathrm{e}^{-4 M \sqrt{x}-4 x}\right)\right]
$$


and is achieved by $\operatorname{Pr}[X=+\sqrt{P}]=\operatorname{Pr}[X=-\sqrt{P}]=1 / 2$. In the low SNR regime, i.e., $\beta \rightarrow 0$, binary coding does not incur significant performance degradation with respect to optimal coding since $C_{A}(\beta)-J_{A}(\beta)=O\left(\beta^{2}\right)$ for every fading distribution [12].

Let $\mathbf{H}$ be the $n \times(n-k)$ parity-check matrix of the binary linear code mapping blocks of $k$ iid (independent and identically distributed) equally likely bits into the length- $n$ codeword $\boldsymbol{c}=\left(c_{1}, \cdots, c_{n}\right)$. Let $\boldsymbol{h}_{j}$ be the $j$-th column of $\mathbf{H}, \boldsymbol{x}=1-2 \boldsymbol{c}$ be the transmitted signal vector and $\boldsymbol{y}=\left(y_{1}, \cdots, y_{n}\right)$ be the channel output vector. The Maximum A Posteriori (MAP) bitwise decoding rule

$$
\widehat{c}_{i}=\arg \max _{a \in\{0,1\}} \operatorname{Pr}\left[c_{i}=a \mid \boldsymbol{y}\right]=\arg \max _{a \in\{0,1\}} \sum_{\boldsymbol{c}: c_{i}=a} \operatorname{Pr}[\boldsymbol{c}, \boldsymbol{y}]
$$

minimizes the probability of coded bit error, where the joint probability $\operatorname{Pr}[\boldsymbol{c}, \boldsymbol{y}]$ factorizes as

$\operatorname{Pr}[\boldsymbol{c}, \boldsymbol{y}]=\frac{1}{2^{k}} \prod_{j=1}^{n-k} \chi_{\left\{\boldsymbol{c} \boldsymbol{h}_{j}=0\right\}} \prod_{i=1}^{n} \frac{1}{\pi N_{0}} \chi_{\left\{x_{i}=1-2 c_{i}\right\}} \mathrm{e}^{-\frac{\left|y_{i}-A_{i} \sqrt{P} x_{i}\right|^{2}}{N_{0}}}$

Fig. 1 shows the portion of the factor graph representing the joint probability in (5) relative to the $i$-th coded bit. The BPSK symbol $x_{i}$ (double circle) is related to the coded bit $c_{i}$ (single circle) via the mapper (double square), which represents the operation $x_{i}=1-2 c_{i}$. The coded bits are related to each other via the check nodes (single square) that represent the code parity-check equations. The symbol $\mu_{i, j}^{(\ell)}$ indicates the loglikelihood ratio (LLR) message to the $i$-th variable node from the $j$-th function node at the $\ell$-th iteration and $\xi_{i, j}^{(\ell)}$ indicates the LLR message from the $i$-th variable node to the $j$-th function node at the $\ell$-th iteration. The variable nodes are numbered from 1 to $n$ while the check nodes from 0 to $n-k$, with the convention that 0 indicates the mapping node. The number of iterations is limited to $\ell_{\max }$.

The message passing decoding algorithm is as follows [5]:

Initialization $(\ell=0)$ : the algorithm starts with the following message "from the channel":

$$
\mu_{i, 0}=4 m_{i} \sqrt{\gamma_{i}}
$$

where $\gamma_{i}=P\left|A_{i}\right|^{2} / N_{0}$ is the instantaneous SNR at the receiver and where $m_{i}=\operatorname{Re}\left\{y_{i} / \sqrt{N_{0}} \mathrm{e}^{-j \angle A_{i}}\right\}$ is the matched-filter output normalized by the standard deviation of the noise.

Variable to check node $\left(\ell=1 \cdots \ell_{\max }\right)$ :

$$
\xi_{i, j}^{(\ell)}=\mu_{i, 0}+\sum_{k \neq j, k>0} \mu_{i, k}^{(\ell-1)}
$$

with the convention $\mu_{i, k}^{(0)}=0$.

Check to variable node $\left(\ell=1 \cdots \ell_{\max }\right)$ :

$$
\mu_{i, j}^{(\ell)}=\log \left(\frac{1+\prod_{v \neq i} \tanh \left(\xi_{v, j}^{(\ell)} / 2\right)}{1-\prod_{v \neq i} \tanh \left(\xi_{v, j}^{(\ell)} / 2\right)}\right)
$$

Termination $\left(\ell=\ell_{\max }\right)$ : the algorithm ends with

$$
d_{i}=\mu_{i, 0}+\sum_{j>0} \mu_{i, j}^{\left(\ell_{\max }\right)}
$$

The MAP rule in (4) is approximated by $\widehat{c}_{i}=\left(1-\operatorname{sign}\left\{d_{i}\right\}\right) / 2$.

The (coded) bit error rate performance of the above algorithm is exactly evaluated, in the limit for large blocklength, by using the density evolution technique [4] or can be visualized by using the EXIT chart method [8]. The former method analytically tracks the evolution of the probability mass function of a randomly chosen message as the decoding progresses, while the latter graphically tracks the evolution of a single parameter related the probability mass function. In Section IV we shall use the (simpler) EXIT chart method to determine the performance of LDPC ensembles used over broadcast channels.

\section{Fading Gaussian Broadcast Channels}

We consider two-user discrete-time complex-valued memoryless BCs without feedback described by

$$
\begin{aligned}
& Y=\left(A X+N_{y}, A\right) \\
& Z=\left(B X+N_{z}, B\right)
\end{aligned}
$$

where $A$ and $B$ are ergodic processes instantaneously known at the corresponding receivers but unknown at the transmitter, $N_{u} \sim \mathcal{N}_{C}\left(0, N_{0}\right)$ is the additive white Gaussian noise at receiver $u \in\{y, z\}$ and $X$ is the transmit signal subject to the power constraint $\mathrm{E}\left[|X|^{2}\right] \leq P$. The channel in (10) models the downlink of wireless systems with delay constraints much larger than the fading coherence time and no feedback channel available.

It is easy to verify that the channel in (10) is not degraded [3] for a general distribution on $(A, B)$. Hence its capacity region is unknown. It has been shown [2] that the so-called "morecapable" region [3] given by

$$
\begin{aligned}
R_{z} & \leq C_{B}(\beta)-C_{B}(\alpha \beta) \\
R_{y}+R_{z} & \leq \min \left\{C_{B}(\beta)-C_{B}(\alpha \beta)+C_{A}(\alpha \beta), C_{A}(\beta)\right\}
\end{aligned}
$$

where $R_{u}$ is the transmission rate for user- $u$ and where $\alpha \in[0,1]$, can always be achieved by the input distribution

$$
\left[\begin{array}{l}
V \\
X
\end{array}\right] \sim \mathcal{N}_{C}\left(\left[\begin{array}{l}
0 \\
0
\end{array}\right], P\left[\begin{array}{cc}
1-\alpha & 1-\alpha \\
1-\alpha & 1
\end{array}\right]\right)
$$

The region in (11) admits the following interpretation. The transmitter independently generates the codeword $V$ for user- $Z$ and the codeword $U$ for user- $Y$ both of unit energy. A fraction $\alpha$ of the available power is assigned to the codeword $U$ and a fraction $(1-\alpha)$ to the codeword $V$. The actual signal sent over the channel is the superposition $X=U \sqrt{\alpha P}+V \sqrt{(1-\alpha) P}$. User-Z decodes its codeword $V$ by treating the codeword $U$ as noise, while user-Y jointly decodes its own message $U$ and the other user message $V$.

As in the single-user case, we are interested in comparing the performance achievable with binary codes with respect to the Gaussian codebook in (12). Similarly to the single-user case, we expect no significant loss in performance from the use of binary codes in the low SNR regime only [12]. In what follows, encoding and decoding are performed as for the Gaussian distribution, however $(U, V)$ is chosen equally likely in $\{0,1\} \times\{0,1\}$. The achievable region with binary input is given by (11) with $C(\beta)$ in (2) replaced by $J(\beta)$ in (3). Fig. 3 and Fig. 4 show the Gaussian region and the binary achievable region, for the unfaded case and for the Rayleigh fading case, respectively. For large SNR the two regions can be far apart.

A multiuser iterative decoding algorithm. The codewords are decoded according to the MAP rule at both receivers. Let $\boldsymbol{h}_{u, j}$ be the $j$-th column of the parity-check matrix of the user- $u$ code, $\boldsymbol{x}=\sqrt{\alpha P}\left(1-2 \boldsymbol{c}_{y}\right)+\sqrt{(1-\alpha) P}\left(1-2 \boldsymbol{c}_{z}\right)$ be transmitted signal (the superposition of the two codewords) and $\boldsymbol{y}$, 
resp. $\boldsymbol{z}$, be the vector of the user-Y, resp. of the user-Z, channel outputs. The MAP estimate of the bit $c_{y, i}$ at the user-Y receiver is

$$
\widehat{c}_{y, i}=\arg \max _{a \in\{0,1\}} \sum_{\boldsymbol{c}_{z}, \boldsymbol{c}_{y}: c_{y, i}=a} \operatorname{Pr}\left[\boldsymbol{c}_{y}, \boldsymbol{c}_{z}, \boldsymbol{y}\right]
$$

where $\operatorname{Pr}\left[\boldsymbol{c}_{y}, \boldsymbol{c}_{z}, \boldsymbol{y}\right]$ is given by

$$
\begin{gathered}
\operatorname{Pr}\left[\boldsymbol{c}_{y}, \boldsymbol{c}_{z}, \boldsymbol{y}\right]=\frac{1}{2^{k_{y}+k_{z}}} \prod_{u=y, z} \prod_{j=1}^{n-k_{u}} \chi_{\left\{\boldsymbol{c}_{u} \boldsymbol{h}_{u, j}=0\right\}} \prod_{i=1}^{n} \frac{1}{\pi N_{0}} . \\
\mathrm{e}^{-\frac{\left|y_{i}-A_{i} x_{i}\right|^{2}}{N_{0}}} \chi_{\left\{x_{i}=\sqrt{\alpha P}\left(1-2 c_{y, i}\right)+\sqrt{(1-\alpha) P}\left(1-2 c_{z, i}\right)\right\}}
\end{gathered}
$$

The decoding of the bit $c_{z, i}$ at the user- $Z$ decoder is completely analogous. Fig. 2 shows the portion of the factor graph representing the joint probability in (14) relative to the $i$-th pair of coded bits $\left(c_{y, i}, c_{z, i}\right)$. The symbols $\xi_{u, i, j}$ (LLR messages from the variable nodes) and $\mu_{u, i, j}$ (LLR messages to the variable nodes) have the meaning explained in Section II with the difference that here the first index refers to the user, the second to the coded bit and the third to the parity-check equation.

At each iteration, messages are exchanged between variable nodes and check nodes on each users' sub-graph (on the left and on the right of the mapper node in Fig. 2) according to (7) and (8), as in the single-user case. However, before the next iteration on the users' subgraphs starts, messages are sent to and received from the mapper node. The message sent to the mapper is

$$
\xi_{u, i, 0}^{(\ell)}=\sum_{c>0} \mu_{u, i, c}^{(\ell)}
$$

while the message sent by the mapper node is [13]

$$
\begin{aligned}
\mu_{y, i, 0}^{(\ell)}= & 4 m_{i} \sqrt{\gamma_{y, i}}+ \\
& \log \left(\frac{\cosh \left(2 m_{i} \sqrt{\gamma_{z, i}}+\xi_{z, i, 0}^{(\ell)} / 2-2 \sqrt{\gamma_{y, i} \gamma_{z, i}}\right)}{\cosh \left(2 m_{i} \sqrt{\gamma_{z, i}}+\xi_{z, i, 0}^{(\ell)} / 2+2 \sqrt{\gamma_{y, i} \gamma_{z, i}}\right)}\right)
\end{aligned}
$$

where $m_{i}=\operatorname{Re}\left\{y_{i} / \sqrt{N_{0}} \mathrm{e}^{-j \angle A_{i}}\right\}$ is the output of the matchedfilter, $x_{u, i}=1-2 c_{u, i}$, and where $\gamma_{u, i}=\alpha_{u} P\left|A_{i}\right|^{2} / N_{0}$ is the instantaneous receive SNR of user- $u$ for $\alpha_{y}=1-\alpha_{z}=\alpha \in[0,1]$. Because of the complete symmetry with respect to the two users, the message $\mu_{z, i, 0}$ equals (16) with all the subscripts $y$ and $z$ interchanged. Similarly, at the user- $Z$ decoder, the mapper node message is computed as in (16) but with $\left(y_{i}, A_{i}\right)$ replaced by $\left(z_{i}, B_{i}\right)$. When user- $Z$ is allotted zero power, i.e., $\gamma_{z, i}=0$, the message in (16) reduces to the "channel observation" in (6) and does not change with the iterations.

The mapper node as a soft interference canceller. The flow of information through the mapper represented by (16) implies that a message referring to a particular user-Y bit is also a function of the other user's bits. Because the bitwise MAP decoder in (13) is inherently a single-user decoder, the presence of the user- $\mathrm{Z}$ codeword in the received signal is never beneficial. We therefore expect that the mapper node somehow performs the cancellation of the interference due to the other user codeword to enhance the reliability of the messages.

The interpretation of the expression in (16) as softinterference cancellation comes from the following observation. The function $f_{t}(x)=\log (\cosh (x+t))-\log (\cosh (x-t))$ for $x \in \mathbb{R}$ is an odd function for any value of the parameter $t$ and, for $|x| \gg 1$, it can be approximated as $f_{t}(x) \approx 2 t \operatorname{sign}(x)$. If the user-Z bit is known with high reliability, i.e, $\xi_{z, i, 0}=x_{z, i}\left|\xi_{z, i, 0}\right|$ with $\left|\xi_{z, i, 0}\right| \gg 1$ (the sign of the message is exact!), then the expression in (16) is approximated by (with an abuse of notation)

$$
\begin{aligned}
\mu_{y, i, 0} \approx & 4 \sqrt{\gamma_{y, i}}\left(\sqrt{\gamma_{y, i}} x_{y, i}+\sqrt{\gamma_{z, i}} x_{z, i}+\mathcal{N}_{R}(0,1 / 2)\right) \\
& -4 \sqrt{\gamma_{y, i} \gamma_{z, i}} x_{z, i} \\
= & 4 \sqrt{\gamma_{y, i}}\left(\sqrt{\gamma_{y, i}} x_{y, i}+\mathcal{N}_{R}(0,1 / 2)\right)
\end{aligned}
$$

which is precisely the matched-filter output $m_{i}$ "cleaned" from the contribution of the user-Z bit $x_{z, i}$.

Moreover, if the expression in (16) is computationally too expensive for implementation in commercial devices, the optimal mapper update rule in (16) can be approximated by $\mu_{y, i, 0}^{\prime}$

$$
\mu_{y, i, 0}^{\prime}=4 \sqrt{\gamma_{y, i}}\left(m_{i}-\sqrt{\gamma_{z, i}} \operatorname{sign}\left\{\xi_{z, i, 0}+4 m_{i} \sqrt{\gamma_{z, i}}\right\}\right)
$$

Interestingly, the above can be interpreted as hard interference cancellation. In fact, the term " $\operatorname{sign}\left\{\xi_{z, i, 0}+4 m_{i} \sqrt{\gamma_{z, i}}\right\}$ " is the "best" hard estimate of the user-Z transmitted bit in a singleuser perspective when the node is given the channel observation $m_{i}$ and the sum of all the messages from the check nodes represented by $\xi_{z, i, 0}$ (recall that the decision rule in the single-user case is given by (9)). Given the hard estimate of the user$\mathrm{Z}$ symbol $x_{z, i}$, the mapper node passes to the user-Y variable node $c_{y, i}$ the received signal $m_{i}$ cleaned from the contribution of $x_{z, i}$. As opposed to the soft interference cancellation in (16), the hard interference cancellation in (18) may suffer from error propagation especially when the user-Z bit reliability is low, i.e., $\left|\xi_{z, i, 0}+4 m_{i} \sqrt{\gamma_{z, i}}\right| \ll 1$.

We are now ready to investigate the bit error rate performance of the derived multi-user iterative message passing decoding algorithm.

\section{Performance Analysis}

The bit error probability after $\ell_{\max }$ decoding iterations is $\mathrm{P}_{\mathrm{bit}}(n)=\operatorname{Pr}\left[\sum_{c \geq 0} \mu_{u, i, c}^{\left(\ell_{\max }\right)} \neq x_{u, i}, u \in\{y, z\}\right]$. Our goal is to find degree distributions $(\lambda, \rho)$ which result in codes with $\mathrm{P}_{\text {bit }}(n) \rightarrow 0$ as $n \rightarrow \infty$. As in the single-user case [4], it suffices to analyze the average performance of the LDPC ensemble and to restrict attention to the cycle-free case. The average performance of an LDPC ensemble can be analyzed either analytically with the density evolution technique [4] or graphically with the EXIT chart method [9]. In the following we shall use the density evolution technique to derive the stability conditions for LDPC codes used over BCs and then we shall turn to the EXIT chart method to optimize the degree distributions.

The stability condition. The stability condition characterizes the behavior of density evolution when, with probability almost one, the density of the LLR messages has a mass point at infinity. We generalize the approach of [4] in order to derive the stability conditions for the pair of codes used on a BC.

It can be shown [13] that the stability condition at the user- $Y$ receiver, where both codewords are jointly decoded, decouples into two independent conditions, one for each code. Reliable decoding is hence possible if

$$
\frac{1}{\lambda_{u}^{\prime}(0) \rho_{u}^{\prime}(1)}>\mathrm{E}_{A}\left[\exp \left\{-\frac{\alpha_{u} P|A|^{2}}{N_{0}}\right\}\right] \quad u \in\{y, z\}
$$

At the user- $Z$ receiver only the codeword for user- $Z$ is decoded by treating the user- $Y$ codeword as noise. It seems unfeasible to 
evaluate in closed form the stability condition when the user- $Y$ codeword is binary. However, a "conservative" condition can be established by considering that user- $Y$ employs a Gaussian code $x_{y, i} \sim \mathcal{N}_{C}(0, \alpha P)$ (recall that Gaussian is the worst case noise [3]). In this case the stability condition becomes

$$
\frac{1}{\lambda_{z}^{\prime}(0) \rho_{z}^{\prime}(1)}>\mathrm{E}_{B}\left[\exp \left\{-\frac{(1-\alpha) P|B|^{2}}{N_{0}+\alpha P|B|^{2}}\right\}\right]
$$

In the following search for good codes, we enforce our candidate degree distributions to fulfill the conditions in (19) and the condition in (20).

The EXIT chart. The EXIT chart method [9] is an effective low-complexity technique of approximately evaluating the asymptotic performance of iterative decoding algorithms. It consists in plotting on the same chart the open-loop inputoutput characteristics of the different components of a concatenated system. Following the approach of [8], we look at LDPC codes as mixtures of repetition codes, one per variable node, concatenated with mixtures of single parity-check codes, one per check nodes.

The variable and check node input-output characteristics are as follows. The mutual information of the outgoing (extrinsic) message at a variable node $I_{E, V}$ is

$I_{E, V} \triangleq \theta\left(I_{A, V}, I_{\mathrm{ch}}\right)=\sum_{i} \lambda_{i} J\left((i-1) J^{-1}\left(I_{A, V}\right)+J^{-1}\left(I_{\mathrm{ch}}\right)\right)$

where $I_{A, V}$ is the mutual information of the incoming (a priori) message at the variable nodes, $I_{\mathrm{ch}}$ is the mutual information of the message from the channel and $J(x)$ is defined in (3). The outgoing (extrinsic) message at a check node is

$$
\left.I_{E, C} \triangleq \eta\left(I_{A, C}\right)=1-\sum_{i} \rho_{i} J\left((i-1) J^{-1}\left(1-I_{A, C}\right)\right)\right)
$$

where $I_{A, C}$ is the mutual information of the incoming (a priori) message at the check node. Because the output of one component becomes the input of the other component, we have $I_{A, C}=I_{E, V}$ and $I_{A, V}=I_{E, C}$. Subject to the approximations inherent in EXIT charts, reliable decoding is possible if

$$
\theta\left(I, I_{\mathrm{ch}}\right)>\eta^{-1}(I), \quad \forall I \in[0,1)
$$

In our multiuser setting, we extend the idea of (23) to track the mutual information of the coded bits in both codewords. The recursion becomes

$$
I_{u}^{(\ell)}=\theta_{u}\left(\eta_{u}\left(I_{u}^{(\ell-1)}\right), \kappa_{a \rightarrow u}\left(I_{a}^{(\ell-1)}\right)\right) \quad a \neq u \in\{y, z\}
$$

where $I_{u}^{(\ell)}$ is the mutual information of the user- $u$ coded bits at iteration $\ell$ and $\kappa_{u \rightarrow a}, u \neq a$, is the mapping node input-output characteristic from user- $u$ to user- $a$. The functions $\theta_{u}$ and $\eta_{u}$ in (24), $u \in\{y, z\}$, are given by (21) and (22), respectively, computed for the degree distributions of the user- $u$ code. Notice that the fixed information $I_{\text {ch }}$ from the channel in (21) has been replaced by the varying information from the mapping node. The mapping node EXIT functions $\kappa_{u \rightarrow a}$ cannot be expressed in closed form and are estimated by Monte-Carlo simulation.

The multiuser equivalent of the condition for reliable decoding in $(23)$ is $I_{u}^{(\ell)} \rightarrow 1$, as $\ell \rightarrow \infty$, for $u \in\{y, z\}$. In our code search we verify this condition to ensure the asymptotic convergence of the iterative decoding algorithm when applied to the candidate LDPC ensemble.
Code optimization. We simplify the code search by first finding a "good" user- $Z$ code and then optimizing the user- $Y$ code, given the found user- $Z$ code. Our optimization algorithm is as follows. We enforce our candidate codes to fulfill the conditions in (19) and in (20). For a fixed degree distribution, we use the EXIT chart method to determine the (approximate) threshold of the corresponding LDPC ensemble. Then, by using the differential evolution algorithm $[5,6,14]$, we modify the found degree distribution and we check whether the new one has either a higher threshold or a higher rate for the same threshold. The discussion on the numerical results concludes the section.

Numerical results. Using the above mentioned code search procedure, we found LDPC codes with (approximate) SNR thresholds close to their Shannon limit for both unfaded and Rayleigh fading channels. For each channel, we considered several values of $\alpha \in[0,1]$ each corresponding to a different power share and hence resulting in a different rate point. Fig. 3 and Fig. 4 show the achievable rate region for binary input and the optimized code rate pairs. The code rate pairs are very close to the closure of the achievable region for both channel models.

We notice (due to space limitations we cannot report all the found codes here, see [13] for an exhaustive list) that codes with higher maximum variable node degree have lower SNR thresholds, as remarked in [5]. Intuition would indeed agree that codes whose bits are involved in more parity-check equations offer greater reliability. Our code search shows that, similarly to what has been observed for erasure channels [14], Gaussian channels [5] and uncorrelated Rayleigh fading Gaussian channels [6], the optimized degree distributions have only few nonzero terms. The variable node degrees tend to be concentrated around the maximum allowable degree, the degree two, and a few other degrees in between. The check node degrees are concentrated around a single degree whose value depends on the average receive SNR. Also, the overall form of the degree distributions are similar for the unfaded and the fading case.

The concentration theorem [4] ensures that for sufficiently large blocklength almost every code in the ensemble will have vanishing probability of error, if the receiver SNR is above the ensemble threshold. This condition was enforced in our code search. However, we are also interested in the code performance for finite blocklengths. As an example, Fig. 5 shows the user- $Y$ bit error rate performance for the following codes

$$
\begin{gathered}
\lambda_{y}(x)=0.16775 x+0.19545 x^{2}+0.22167 x^{7} \\
+0.08149 x^{8}+0.33366 x^{49} \\
\rho_{y}(x)=0.12033 x^{9}+0.87967 x^{10} \\
\lambda_{z}(x)=0.13259 x+0.32749 x^{2}+0.26180 x^{9} \\
+0.00377 x^{10}+0.00038 x^{48}+0.27398 x^{49} \\
\rho_{z}(x)=0.45642 x^{7}+0.54356 x^{8}+0.00002 x^{12}
\end{gathered}
$$

with blocklength $n=10^{3}$ and $n=10^{4}$. The degree distributions were optimized for an unfaded channel with $|B|^{2} P / N_{0}=$ $3.871 \mathrm{~dB},|A|^{2} P / N_{0}=5.059 \mathrm{~dB}$ and $\alpha=0.35$. The design rate is 0.522 . The dashed line shows the asymptotic threshold for this ensemble pair. We notice that the threshold effect is already quite pronounced at a blocklength of $10^{4}$.

\section{Conclusions}

In this work we studied the performance of LDPC codes on Gaussian BCs with fading. We showed that good degree distribution pairs can be found to approach the Shannon limit for 
binary constrained inputs. We computed the stability conditions the pair of codes must satisfy in order to ensure vanishing probability of error for large blocklength and infinite iterations. We enforced this condition when optimizing the degree distributions by using both the EXIT chart method and the differential evolution algorithm. We showed that the optimized code operate at about $1 \mathrm{~dB}$ of their threshold for finite blocklength.

We derived a multiuser iterative message passing decoding algorithm for joint decoding of the user codewords at the best receiver and for single-user decoding at the worst receiver. In applying the sum-product update rule to deriving the joint message passing decoding algorithm, we found that the message update rule at the mapping node linking the users' codes is equivalent to soft interference cancellation. Interesting, a low complexity implementation of the optimal update rule at the mapper is shown to be equivalent to hard interference cancellation.

Acknowledgments. The authors would like to thank Abdelaziz Amraoui for helpful discussions.

\section{REFERENCES}

[1] T. M. Cover, "Broadcast Channels," IEEE Trans. on Inform. Theory, vol. 18, pp. 214, January 1972.

[2] D. Tuninetti, and S. Shamai, "Fading Gaussian broadcast channels with state information at the receivers," in DIMACS Workshop on Network Information Theory,

3] T. M. Cover and J. A. Thomas, Elements of Information Theory. New York: Wiley, 1991.

[4] T. J. Richardson and R. L. Urbanke, "The capacity of low-density parity-check codes under message-passing decoding," IEEE Trans. on Inform. Theory, vol. 47, pp. 599-618, February 2001.

[5] T. J. Richardson, M. A. Shokrollahi, and R. L. Urbanke, "Design of capacityapproadching irregular low-density parity-check codes," IEEE Trans. on Inform. Theapproadching irregular low-density parity-ch
ory, vol. 47, pp. 619-637, February 2001.

[6] J. Hou, P. H. Siegel, and L. B. Milstein, "Performance analysis and code optimization of low-density parity-check codes on Rayleigh fading chan
J. on Selected Areas in Communications, vol. 19, pp. 924-934, May 2001.

[7] A. Amraoui, S. Dusad, and R. Urbanke, "Achieving general points in the 2-user coding," in Proc. ISIT 2002, (Lausanne, Switzerland), p. 334, July 2002

[8] S. ten Brink, G. Kramer, and A. Ashikhmin, "Design of low-density parity-check codes for modulation and detection," IEEE Trans. Commun. to appear April 2004

9] S. ten Brink, "Convergence of iterative decoding," Electronics Letters, vol. 35, pp. 806-808, May 1999.

[10] R. G. Gallager, Low-Density Parity-Check Codes. Cambridge, MA: MIT Press, 1963.

[11] B. J. F. Frank, R. Kschischang and H.-A. Loeliger, "Factor graphs and the sumproduct algorithm," IEEE Trans. on Inform. Theory, vol. 47, pp. 498-519, February 2001.

[12] S. Verdú, G. Caire, and D. Tuninetti, "Is TDMA optimal in the low-power regime?," in Proc. ISIT 2002, (Lausanne, Switzerland), p. 193, July 2002

[13] P. Berlin and D. Tuninetti, "LDPC codes for Gaussian broadcast channels," submitted to IEEE Trans. on Inform. Theory, February 2004. [14] A. Shokrollahi and R. Storn, "Design of efficient erasure codes with differential

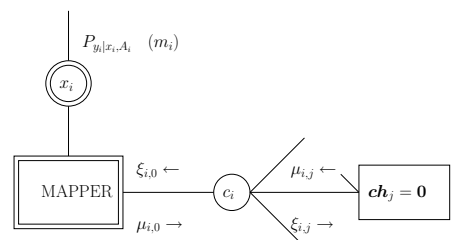

Fig. 1: Section of the factor graph referring to the $i$-th coded bit.

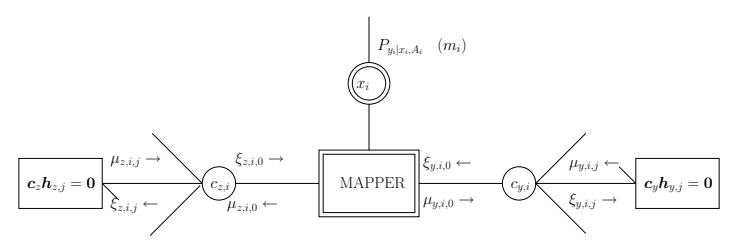

Fig. 2: Section of the factor graph referring to the $i$-th pair of coded bits.

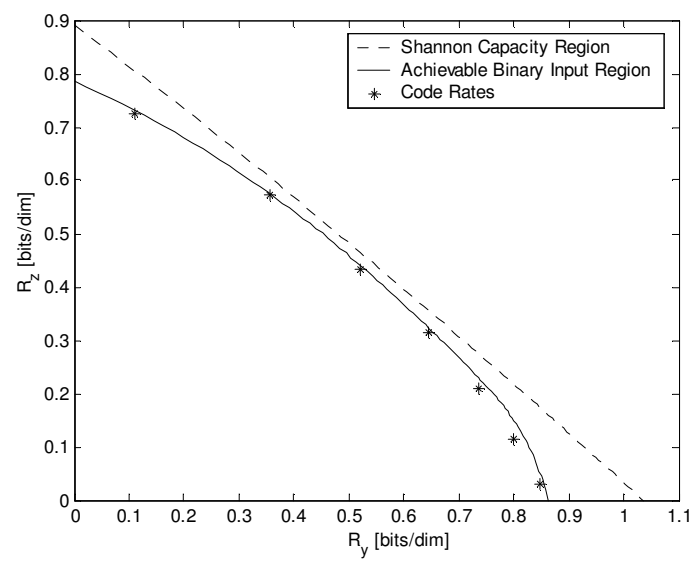

Fig. 3: Achievable regions and rates of the LDPC codes found for the unfaded $\mathrm{BC}$ with $|A|^{2} P / N_{0}=5.059 \mathrm{~dB}$ and $|B|^{2} P / N_{0}=3.871 \mathrm{~dB}$.

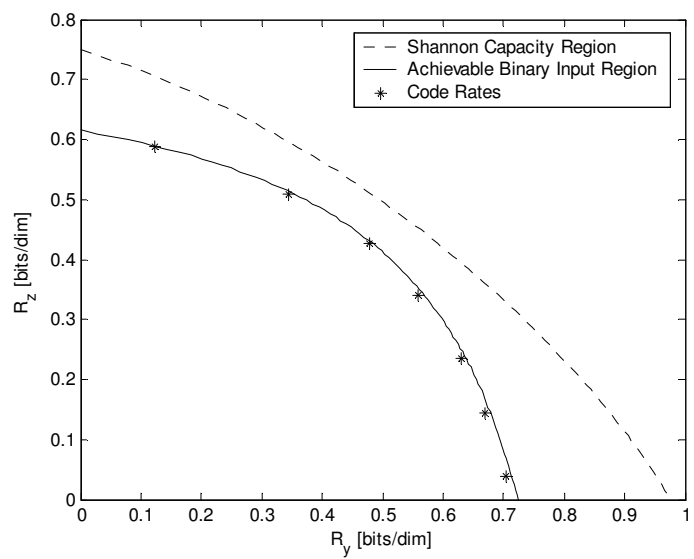

Fig. 4: Achievable regions and rates of the LDPC codes found for the Rayleigh fading $\mathrm{BC}$ with $\mathrm{E}\left[|A|^{2}\right] P / N_{0}=3.098 \mathrm{~dB}$ and $\mathrm{E}\left[|B|^{2}\right] P / N_{0}=0.915 \mathrm{~dB}$.

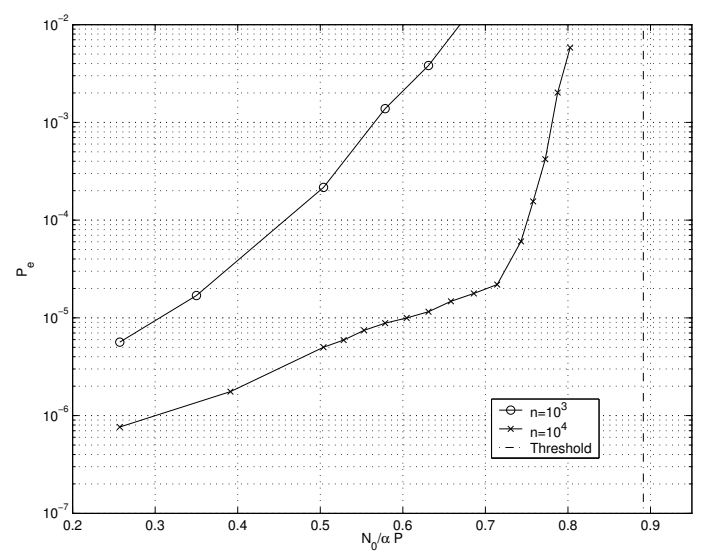

Fig. 5: User-Y BER (over systematic bits only) for joint decoding of the codes in (25)-(28) for finite blocklength. 